\title{
Foot Ulcers Caused by Rheumatoid Vasculitis in a Patient with Rheumatoid Arthritis Undergoing Etanercept Treatment
}

\author{
Takamasa Murosaki, Takao Nagashima, Yoko Aoki, Yukiko Imai, \\ Masahiro Iwamoto and Seiji Minota
}

\begin{abstract}
A 59-year-old woman with a 10-year history of rheumatoid arthritis (RA) presented with chronic ulcers on both feet while undergoing treatment with etanercept. Rheumatoid vasculitis (RV) was diagnosed, and the patient was treated with immunosuppressant drugs and skin grafting. Although anti-tumor necrosis factor (TNF) agents are known to induce vasculitis, vasculitis can also be caused by active RA. Accordingly, the cause of vasculitis in RA patients receiving anti-TNF therapy must be evaluated carefully.
\end{abstract}

Key words: anti-tumor necrosis factor therapy, leukocytoclastic vasculitis, rheumatoid arthritis, rheumatoid vasculitis

(Intern Med 51: 3181-3183, 2012)

(DOI: 10.2169/internalmedicine.51.8424)

\section{Introduction}

Foot ulcers are common in patients with rheumatoid arthritis (RA). The etiology is often multifactorial, including vasculitis, relative arterial insufficiency, venous insufficiency, dependent edema, chronic glucocorticoid use, repeated trauma and restricted ankle movement (1). Cutaneous vasculitis is one of the major extra-articular manifestations of RA and is often called rheumatoid vasculitis (RV) (1). On the other hand, leukocytoclastic vasculitis (LCV) of the skin is a known adverse reaction to anti-tumor necrosis factor (TNF) therapy (2). We herein present the case of a patient with RA who developed bilateral foot ulcers while undergoing etanercept treatment. Although LCV was found histologically, we diagnosed the patient with RV rather than drug (etanercept)induced vasculitis.

\section{Case Report}

A 59-year-old woman with a 10-year history of RA was referred to our hospital for treatment of foot ulcers. She had initially taken prednisolone and several disease modifying anti-rheumatic drugs (DMARDs), including methotrexate; however, all DMARDs were ineffective. Four years earlier, treatment with etanercept ( $25 \mathrm{mg}$ twice a week) had been started. A good response was observed for six months; however, the efficacy of the treatment gradually decreased. One year before presentation, refractory ulcers developed on both feet and gradually became enlarged despite the use of topical treatments. Etanercept was discontinued one month before hospitalization because the ulcers were thought to have been induced by the drug; however, no improvement was observed after discontinuation.

The ulcers measured $14 \times 6 \mathrm{~cm}$ on the left foot and $5 \times 4$ $\mathrm{cm}$ on the right foot (Fig. 1). No palpable purpura or apparent peripheral neuropathy were seen. The disease activity score 28 value (based on the erythrocyte sedimentation rate) was 6.01. The hematology test results were as follows: a leukocyte count of $11,400 / \mu \mathrm{L}$ (neutrophils $96 \%$ and lymphocytes $2.6 \%$ ), a hemoglobin level of $9.7 \mathrm{~g} / \mathrm{dL}$ and a platelet count of $36.8 \times 10^{4} / \mu \mathrm{L}$. The serological findings were as follows: C-reactive protein (CRP): $9.0 \mathrm{mg} / \mathrm{dL}$; complement: C3: $65 \mathrm{mg} / \mathrm{dL}$ (normal: 86-160), C4: $6 \mathrm{mg} / \mathrm{dL}$ (17-45), CH50: $20.5 \mathrm{U} / \mathrm{mL}$ (25-48); and serum immune complexes: $4.4 \mu \mathrm{g} / \mathrm{mL}(<3.0)$. The rheumatoid factor $(\mathrm{RF})$ value was 1 : 


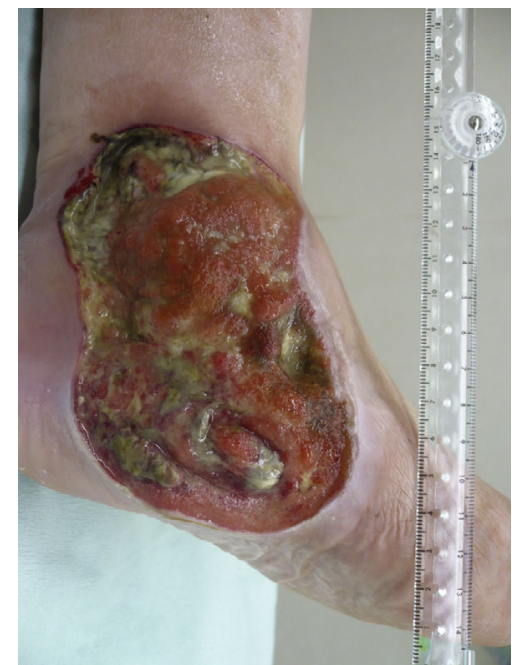

Figure 1. A deep, giant ulcer on the left medial malleolus with necrotic tissue at the periphery.

$5,120(<1: 40)$ and the level of anti-cyclic citrullinated peptide (CCP) antibody was $400 \mathrm{IU} / \mathrm{mL}(<4.5)$. Tests for antinuclear antibody, lupus anticoagulant, anti-cardiolipin antibodies, anti- $\beta 2$ glycoprotein I antibody and antineutrophil cytoplasmic antibodies were all negative. A swab culture of the ulcers revealed several bacteria. A skin biopsy specimen obtained from the ulcer margin demonstrated leukocytoclastic vasculitis (LCV) along with fibrinoid necrosis (Fig. 2). Radiographs of the hands showed mutilans deformity. The ulcers had developed three years after the start of etanercept, and no improvement was observed after discontinuation of the drug. In view of the time course, a diagnosis of antiTNF-induced ulcers was unlikely. From these findings, a diagnosis of RV was thus made.

The patient was treated with intravenous meropenem $(1 \mathrm{~g} /$ day) along with rigorous debridement and maggot application. After the infection subsided, treatment with oral cyclophosphamide $(2 \mathrm{mg} / \mathrm{kg})$ was started. However, the patient's arthritis flared and the CRP level increased from 5.6 to 21.7 $\mathrm{mg} / \mathrm{dL}$. Therefore, the dose of prednisolone was increased from $14 \mathrm{mg} /$ day to $40 \mathrm{mg} / \mathrm{day}(1 \mathrm{mg} / \mathrm{kg})$. Thereafter, both ulcer beds gradually filled with new granulation tissue; however, the lesions did not change in size. Skin grafting was performed six weeks later and both ulcers healed completely four months after grafting.

\section{Discussion}

Our patient developed RV while undergoing etanercept treatment in association with a flare-up of RA. The vasculitic origin of the ulcers was confirmed on a histopathological examination and the administration of strong immunosuppressive therapy was required. The patient had a long history of RA with multiple DMARD use, high serum levels of $\mathrm{RF}$ and anti-CCP antibodies, low levels of complement components and positivity for immune complexes. All of these findings are consistent with the characteristics of

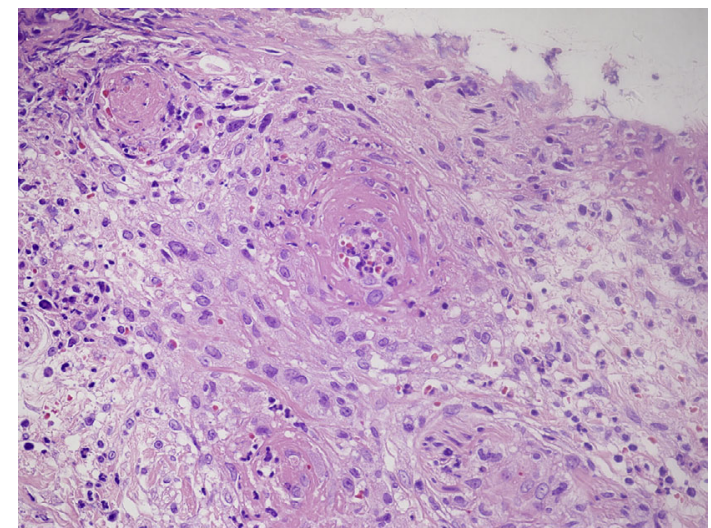

Figure 2. Fibrinoid necrosis with perivascular neutrophil infiltration, granulomatous changes and nuclear debris (Hematoxylin and Eosin staining, $\times \mathbf{4 0 0}$ ).

RV (3, 4). Therefore, etanercept therapy was not strong enough to prevent the development of RV in this patient.

There have been several case reports of anti-TNF therapy being effective for RV (5-10). In one case series, six of nine patients with RV who had received prior treatment with cyclophosphamide and high-dose glucocorticoids achieved remission after six months of infliximab or etanercept treatment (9). The case of a patient with leg ulcers who was successfully treated with adalimumab has also been reported (8). In RA patients with ulcers of the extremities, anti-TNF therapy has been reported to accelerate ulcer healing (11).

Although development of LCV during anti-TNF therapy has been widely reported $(2,12)$, vasculitis is not usually associated with ulcerated lesions, and more than half of affected patients have palpable purpura (12). The median time from the start of etanercept therapy to the development of vasculitis is reported to be 38 weeks (2), and two thirds of patients improve after stopping anti-TNF treatment (2). Therefore, the vasculitis observed in our patient was unlikely to be drug-induced because the foot ulcers developed three years after the start of etanercept therapy and the onset coincided with worsening of the arthritis. Additionally, there were no purpura and the ulcers were deep, suggesting that larger arteries were involved.

The etiology of vasculitis in RA patients on anti-TNF therapy is probably two-fold, involving both anti-TNFinduced vasculitis and anti-TNF-resistant RV. The relationship between vasculitis and treatment is unclear in some reported cases, such as in patients with skin lesions that persist despite drug discontinuation, patients without recurrence of vasculitis after drug reintroduction and patients in whom vasculitis resolves despite the continuance of drug therapy $(2,12)$. These cases are somewhat atypical for druginduced vasculitis. It has been reported that vasculitis occurs more often in patients with RA receiving anti-TNF therapy than in RA patients not receiving anti-TNF therapy (13). However, patients receiving anti-TNF therapy have higher baseline disease activity and more extra-articular manifesta- 
tions that occur before vasculitis than those not receiving anti-TNF therapy. Only two of six reported patients with vasculitis receiving anti-TNF therapy showed spontaneous resolution after the withdrawal of treatment. Patients with refractory RA often develop RV and are also more likely to be treated with biological agents, which may partially account for the higher incidence of vasculitis in patients receiving anti-TNF therapy.

In conclusion, vasculitic skin ulcers caused by RV can develop in RA patients even during the administration of antiTNF therapy. If discontinuation of anti-TNF agents does not improve the ulcers swiftly, the possibility of RV should be carefully considered and evidence to support a diagnosis of RV should therefore be aggressively evaluated.

\section{The authors state that they have no Conflict of Interest (COI).}

\section{References}

1. Turesson C, Jacobsson LT. Epidemiology of extra-articular manifestations in rheumatoid arthritis. Scand J Rheumatol 33: 65-72, 2004.

2. Mohan N, Edwards ET, Cupps TR, et al. Leukocytoclastic vasculitis associated with tumor necrosis factor-alpha blocking agents. J Rheumatol 31: 1955-1958, 2004.

3. Turesson C, Matteson EL. Vasculitis in rheumatoid arthritis. Curr Opin Rheumatol 21: 35-40, 2009.

4. Voskuyl AE, Zwinderman AH, Westedt ML, Vandenbroucke JP, Breedveld FC, Hazes JM. Factors associated with the development of vasculitis in rheumatoid arthritis: results of a case-control study. Ann Rheum Dis 55: 190-192, 1996.

5. Bartolucci $P$, Ramanoelina J, Cohen $P$, et al. Efficacy of the antiTNF-alpha antibody infliximab against refractory systemic vasculitides: an open pilot study on 10 patients. Rheumatology (Oxford) 41: 1126-1132, 2002.

6. Unger L, Kayser M, Nusslein HG. Successful treatment of severe rheumatoid vasculitis by infliximab. Ann Rheum Dis 62: 587-588, 2003.

7. Benucci M, Li Gobbi F, Saviola G, Manfredi M. Improved rheumatoid digital vasculitis in a patient treated with $\mathrm{TNF} \alpha$ agent blocking (infliximab). Rheumatol Int 28: 1253-1255, 2008.

8. Hirche D, Rubbert A, Lunau L, Krieg T, Eming SA. Successful treatment of refractory rheumatoid arthritis-associated leg ulcerations with adalimumab. Br J Dermatol 152: 1062-1064, 2005.

9. Puechal X, Miceli-Richard C, Mejjad O, et al. Anti-tumour necrosis factor treatment in patients with refractory systemic vasculitis associated with rheumatoid arthritis. Ann Rheum Dis 67: 880-884, 2008.

10. Matthews C, Fitzgerald O. Seropositive erosive rheumatoid arthritis (RA). Rheumatology (Oxford) 45: 1100, 2006.

11. Shanmugam VK, DeMaria DM, Attinger CE. Lower extremity ulcers in rheumatoid arthritis: features and response to immunosuppression. Clin Rheumatol 30: 849-853, 2011.

12. Ramos-Casals M, Brito-Zeron P, Munoz S, et al. Autoimmune diseases induced by TNF-targeted therapies: analysis of 233 cases. Medicine (Baltimore) 86: 242-251, 2007.

13. Guignard S, Gossec L, Bandinelli F, Dougados M. Comparison of the clinical characteristics of vasculitis occurring during anti-tumor necrosis factor treatment or not in rheumatoid arthritis patients. A systematic review of 2707 patients, 18 vasculitis. Clin Exp Rheumatol 26: S23-S29, 2008.

(C) 2012 The Japanese Society of Internal Medicine http://www.naika.or.jp/imonline/index.html 